\title{
A platform for testing the performance of metaheuristics solving the energy resource management problem in smart grids
}

\author{
Fernando Lezama ${ }^{1 *}$, Joao Soares ${ }^{1}$ and Zita Vale ${ }^{2}$ \\ From The 7th DACH+ Conference on Energy Informatics \\ Oldenburg, Germany. 11-12 October 2018
}

\footnotetext{
* Correspondence: flzcl@isep.ipp.pt 1 GECAD Research Group, School of Engineering, Polytechnic of Porto, Porto, Portugal

Full list of author information is available at the end of the article
}

\begin{abstract}
Due to the importance of the energy resource management (ERM) in the energy community, several mathematical formulations have been successfully proposed to solve the problem. However, due to the very dynamic evolution of power systems and the transformation of electrical grids, mainly due to the development of smart grid technologies, traditional formulations, which were designed for an entirely different scenario, sometimes cannot deal with the problem efficiently. It is in those situations, where traditional approaches fail, that modern metaheuristic optimizers have demonstrated been a potent tool to face such challenges. In this paper, we present "Meta-ERM", a MATLABC platform designed to assess the performance of modern metaheuristics when solving the ERM problem.
\end{abstract}

Keywords: Energy resource management, Metaheuristics, Optimization, Simulation

\section{Introduction}

The energy resource management (ERM) has been considered one of the most complicated optimization problems in power systems due to its combinatorial nature, nonlinearities and a large number of energy resources which leads to high dimensionality and highly constrained problems (Soares et al. 2016; Soares et al. 2018). Therefore, adequate optimization frameworks and extensive analysis of case studies are a crucial part of the new paradigm brought the by the smart grid operation.

One of the main challenges in the new power system paradigm is that advanced (and occasionally expensive) infrastructure is required to study, validate, and provide accurate projections about how efficient a new control algorithm can be in real implementations. Besides, when a new approach to solve the ERM problem is proposed, it is desired to include a broad range of aspects that can impact its performance (i.e., scalability, uncertainty, memory requirements, operational costs) before real implementation (Di Somma et al. 2018). In this situation, ERM simulation platforms can provide a more accurate perception of the impact of different

(c) The Author(s). 2018 Open Access This article is distributed under the terms of the Creative Commons Attribution 4.0 International License (http://creativecommons.org/licenses/by/4.0/), which permits unrestricted use, distribution, and reproduction in any medium, provided you give appropriate credit to the original author(s) and the source, provide a link to the Creative Commons license, and indicate if changes were made. 
aspects of a new solution before the actual execution takes place (Yan et al. 2013; Cheng et al. 2017; Lezama et al. 2018a).

In this paper, we present "Meta-ERM", a MATLAB@ platform for solving energy resource management problems using stochastic optimization techniques. The platform allows the optimization of large-scale centralized day-ahead scheduling problems with the use of modern metaheuristic algorithms (e.g., PSO, DE, GA, SA, ABC, and others) (Talbi 2009; Faia et al. 2017). The simulation tool has been designed to easy test and assess the performance of any existing metaheuristic.

\section{Description of the energy resource management problem}

Meta-ERM platform solves the energy resource management problem faced by an aggregator (see Fig. 1) aiming at procuring energy needs from distributed resources and the electricity market. The aggregator looks for the minimization of operational costs while making revenues from selling energy in available electricity markets. Moreover, it may use its assets, e.g., energy storage systems (ESS), to supply the load demand. The main idea is that the optimization software performs the energy resource scheduling of the dedicated resources for the $24 \mathrm{~h}$ of the following day (i.e., day-ahead scheduling).

Even with state-of-the-art solvers, it is somehow difficult to provide efficient solutions to some of the scenarios that can be faced in the context of large-scale ERM problems. Therefore, the final purpose of the Meta-ERM platform is to be used for the assessment and comparison of stochastic optimization algorithms (e.g., PSO or DE) to mitigate the exponential execution time and large memory requirements using traditional mathematical tools. Moreover, due to the stochastic nature of these optimizers, an assessment tool is of particular interest for the scientific community working in the development of such approaches.

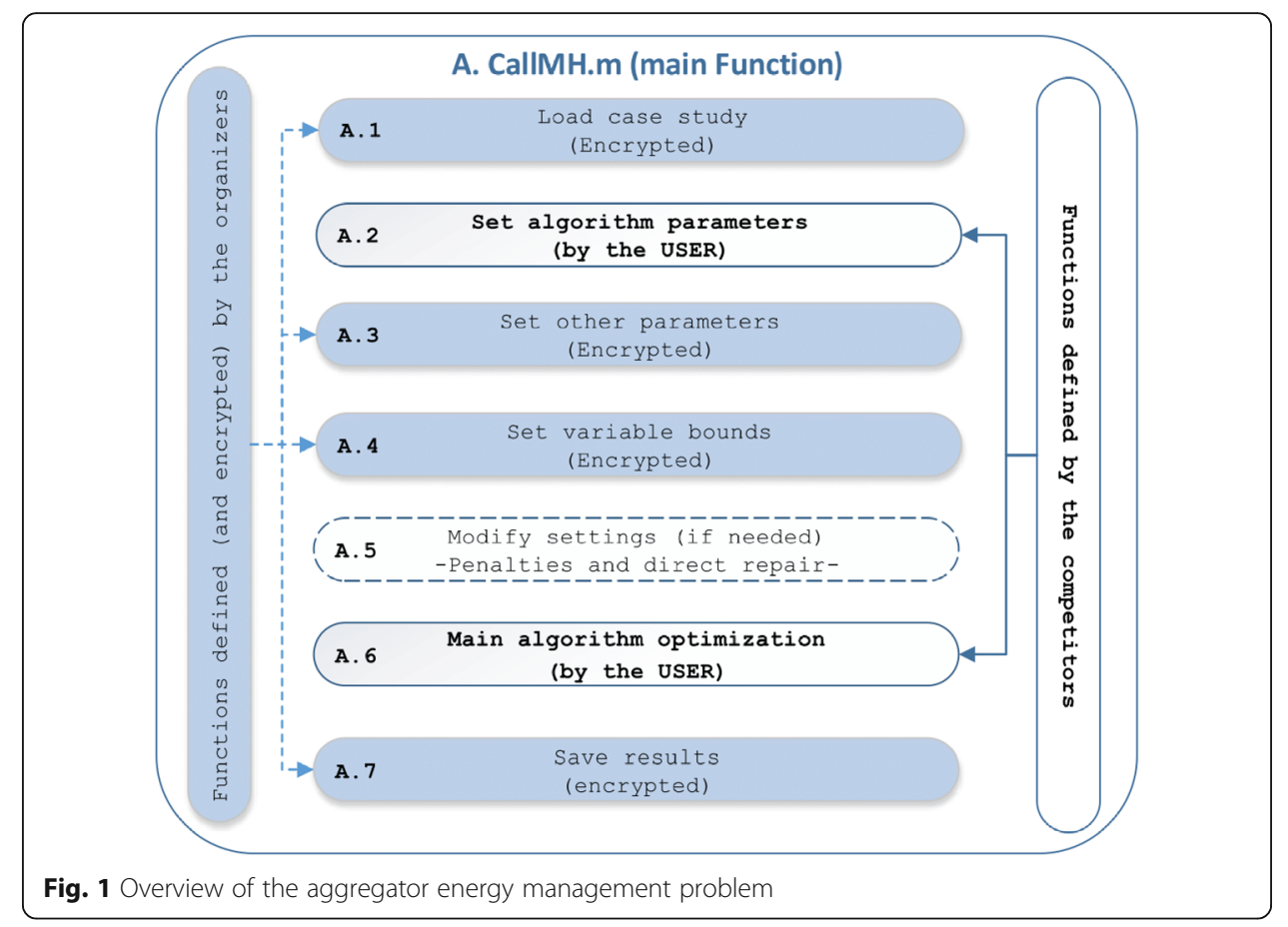




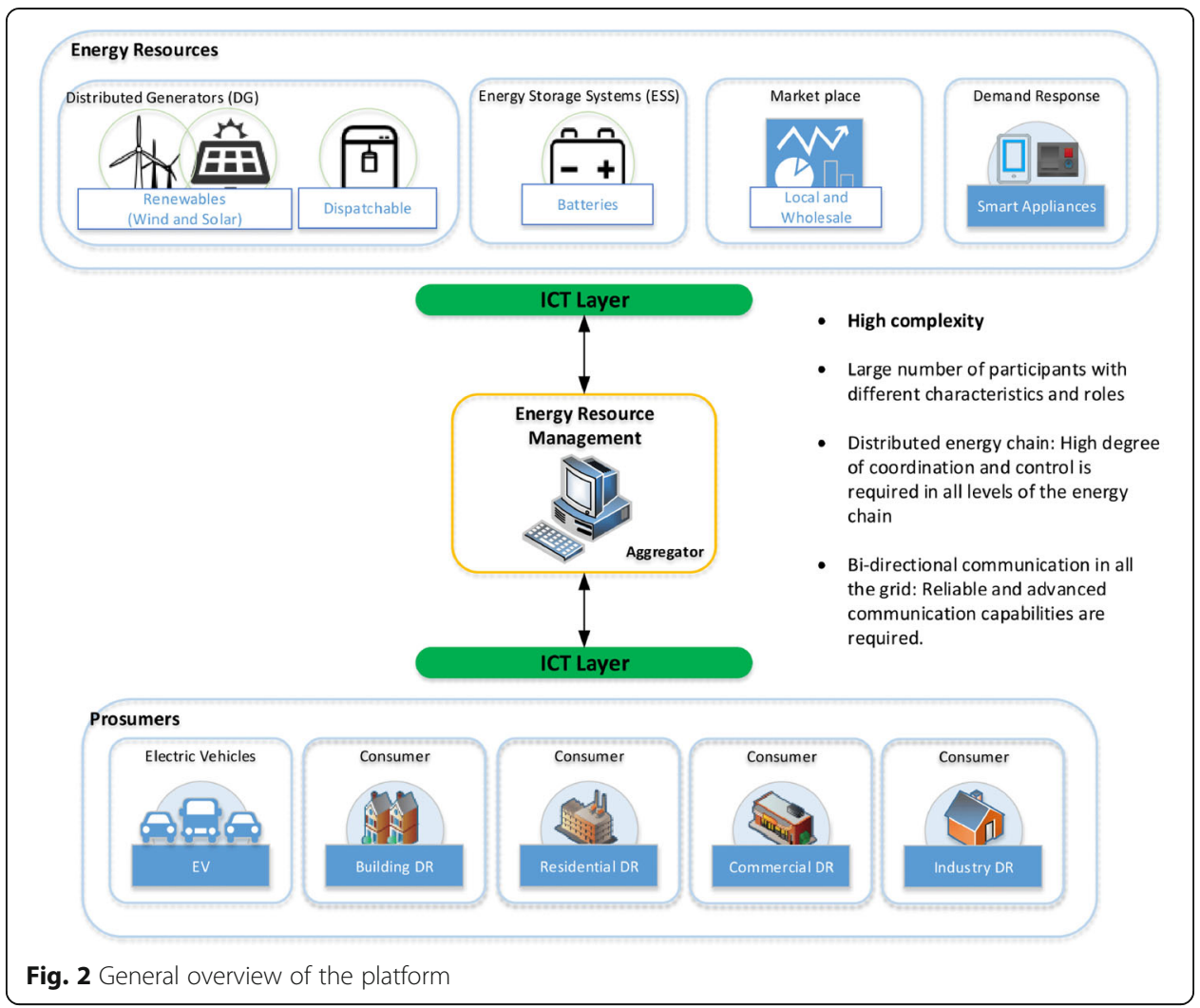

\section{Meta-ERM platform}

The "Meta-ERM" platform was designed using building-blocks that represent functions (i.e., scripts) with specific targets. The simulation platform has been implemented in MATLABC 2016 64-bit. As shown in Fig. 2, some scripts can be encrypted (blue color in the figure), in case the information concerning a specific function cannot be disclosed. Also, with this capability, users interested in testing new algorithms can be focused on modifying (or implementing from scratch) only two scripts, namely:

i) one script for setting the parameters required by their algorithm (A.2).

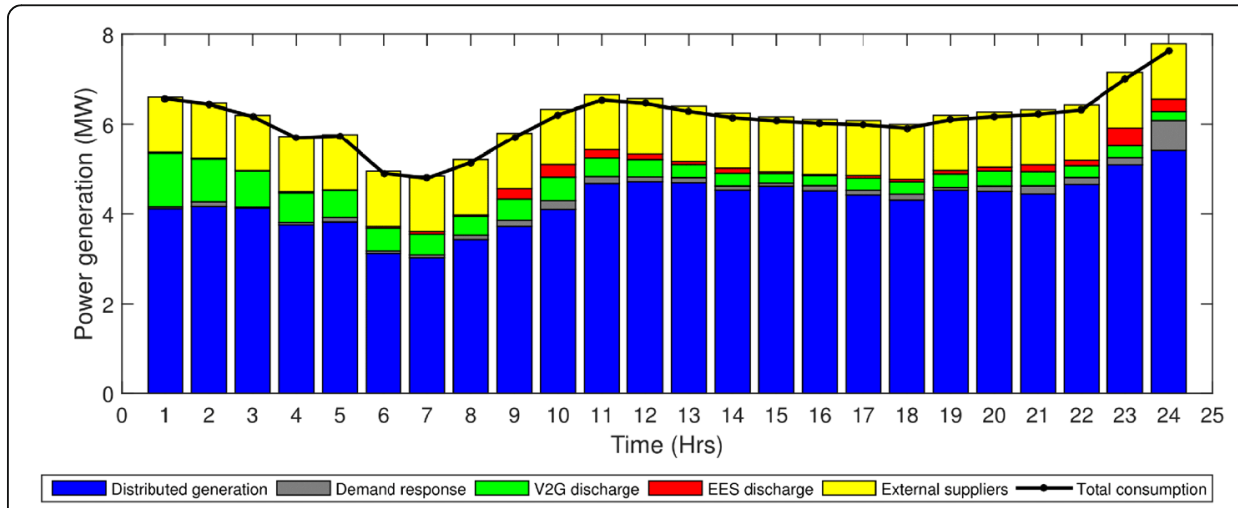

Fig. 3 Plots of power scheduling generated with Meta-ERM platform 


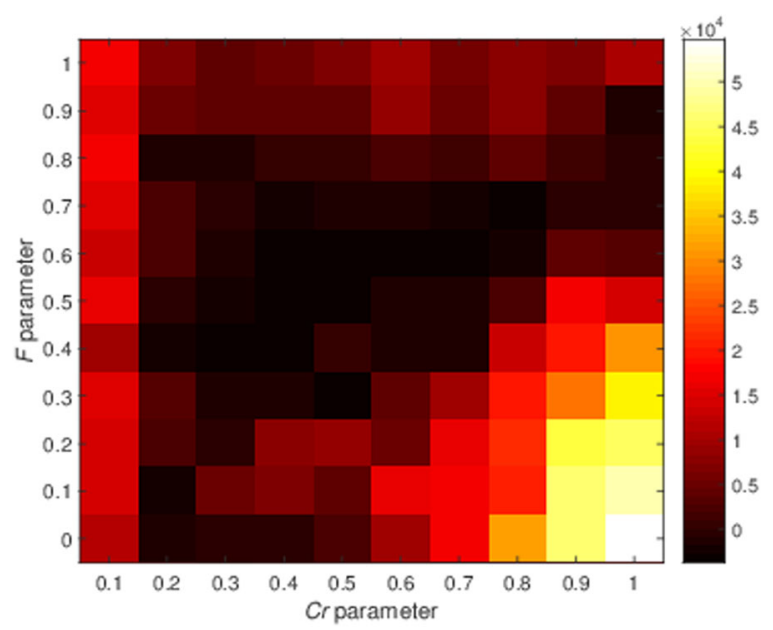

Fig. 4 Heatmaps of metaheuristic parameter tuning generated with Meta-ERM platform

ii) a second script for the implementation of their proposed solution method (A.6). Some applications of the platform can be found in (Soares et al. 2016; Lezama et al. 2018b).

\section{Performance statistics and plot capabilities}

The simulation platform is already configured to allow users choose from a wide variate of case studies (e.g., buildings or microgrids with resources from renewables to EVs) and a comprehensive set of evolutionary algorithms including PSO, DE (in different variants), QPSO, ABC, GA, and others.

Since the performance of stochastic optimizers typically requires a statistical assessment of the provided results, "Meta-ERM" has the capability of configuring many runs and provide statistical indicators (e.g., mean fitness value, execution time, convergence rate) to determine the statistical relevance of the results. This

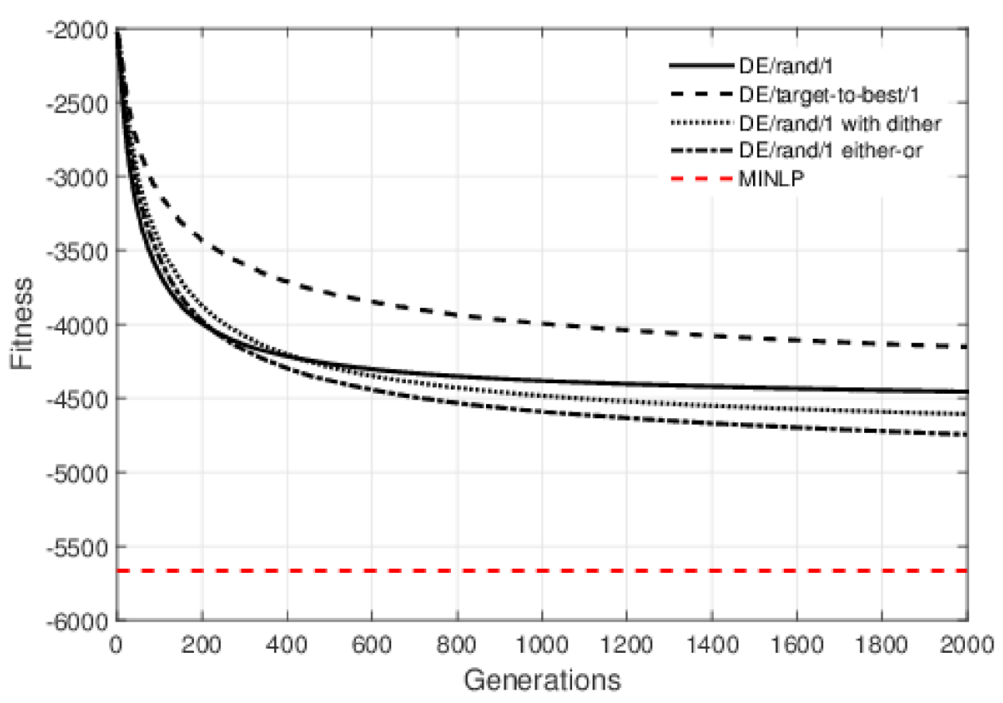

Fig. 5 Plot of algorithms' convergence generated with Meta-ERM platform 


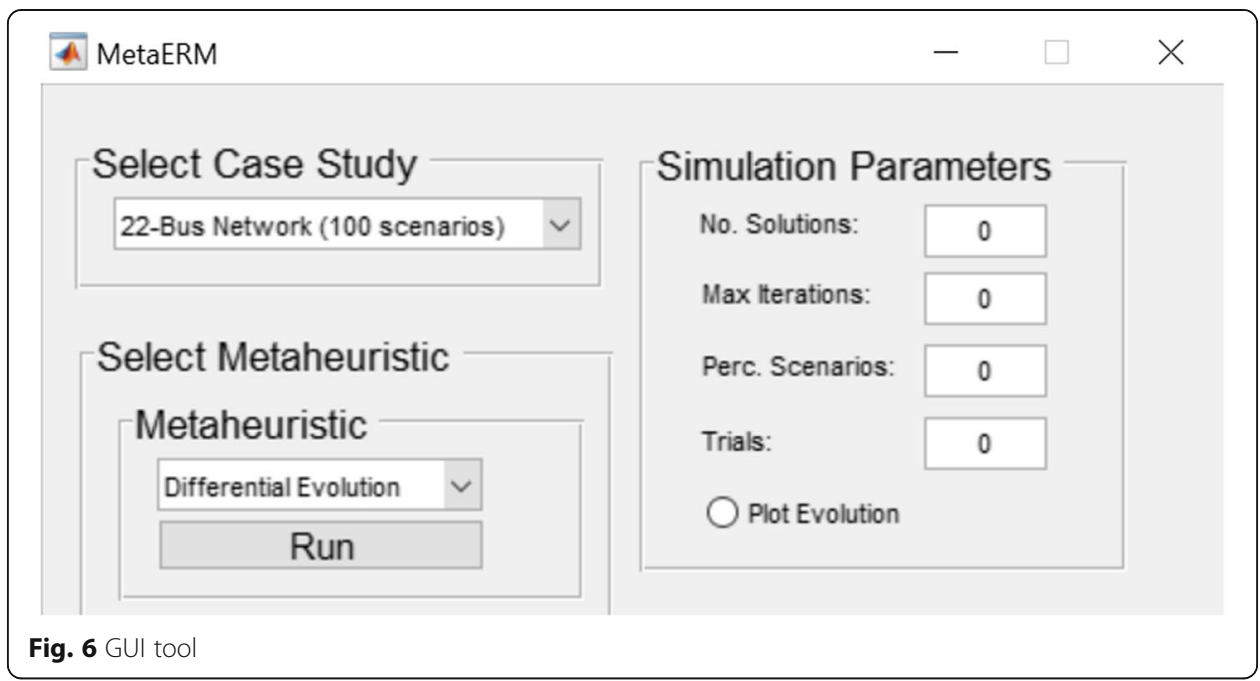

data is provided in the form of output Tables (not shown in the paper for space limitation).

Additionally, the platform is also able to provide a set of different plots for analysis of the solutions. The user can easily configure the type of plots they want to recover from a simulation. The plots are not only limited to the analysis of the quality of the solution provided by an algorithm regarding the scheduling of resources (see, for instance, Fig. 3, but also to the insights concerning the performance of an algorithm (or a group of algorithms) in terms of fitness quality, convergence, robustness or other indicators (see, for instance, Figs. 4 and 5).

\section{Graphical user interface}

A graphical user interface (GUI) is currently under development for the "Meta-ERM" platform (see Fig. 6). Earlier versions of this platform, without GUI, have demonstrated their value for the scientific community through algorithm competition initiatives launched at major and prominent international events (cf. IEEE PES GM 2017 (Lezama et al. 2018b) and WCCI 2018 (Lezama et al. 2017)). The GUI is intended to ease the process of testing algorithms. Through simple menus, the user can choose from over a wide range of case studies/algorithms and tune some global parameters. The GUI interface can be updated periodically, adding functionalities, case studies and algorithms on the go.

\section{Final remarks}

"Meta-ERM" platform is a tool designed to test and validate the performance of metaheuristics when solving the ERM problem in smart grids. Different functionalities are provided by the platform, for instance, different case studies can be selected, a set of popular metaheuristics is available for comparison and performance validation, capabilities for statistical analysis and plot functionalities, among other options. Moreover, because of the building-block structure, new approaches can be implemented and integrated into the platform easily, allowing to expand the state of the art regarding metaheuristic optimization on power systems. 


\section{Abbreviations}

ABC: Artificial Bee Colony; DE: Differential Evolution; ERM: Energy Resource Management; ESS: Energy Storage System; EVs: Electric Vehicles; GA: Genetic Algorithm; GM: General Meeting; GUI: Graphical User Interface; IEEE: Institute of Electrical and Electronics Engineers; PES: Power Energy Society; PSO: Particle Swarm Optimization; QPSO: Quantum Particle Swarm Optimization; SA: Simulate Annealing; WCCI: World Congress on Computational Intelligence

\section{Acknowledgements}

We thank the support of the IEEE PES Working Group on Modern Heuristic Optimization (WGMHO) and the IEEE CIS Task Force on Computational Intelligence in the Energy Domain in the realization of competition initiatives. We would also like to show our gratitude to the Research Group on Intelligent Engineering and Computing for Advanced Innovation and Development (GECAD).

This work has received funding from the European Union's Horizon 2020 research and innovation programme under the Marie Sklodowska-Curie grant agreement No. 641794 project DREAM-GO.

\section{Funding}

This work has received funding from the European Union's Horizon 2020 research and innovation programme under the Marie Sklodowska-Curie grant agreement No. 641794 project DREAM-GO.

\section{Availability of data and materials}

The platform has been used in optimization competition initiatives launched at major and prominent international events. The guidelines and codes are available on-line in:

IEEE PES GM2007 (testbed 2).

http://sites.ieee.org/psace-mho/2017-smart-grid-operation-problems-competition-panel/ WCCI 2018 (Evolutionary Computation in Uncertain Environments: A Smart Grid Application). http://www.gecad.isep.ipp.pt/WCCI2018-SG-COMPETITION/

\section{About this supplement}

This article has been published as part of Energy Informatics Volume 1 Supplement 1, 2018: Proceedings of the 7th $\mathrm{DACH}+$ Conference on Energy Informatics. The full contents of the supplement are available online at https:// energyinformatics.springeropen.com/articles/supplements/volume-1-supplement-1.

\section{Authors' contributions}

All the authors have read and approved the final manuscript. All authors contributed equally to this work.

\section{Competing interests}

The authors declare that they have no competing interests.

\section{Publisher's Note}

Springer Nature remains neutral with regard to jurisdictional claims in published maps and institutional affiliations.

\section{Author details}

${ }^{1}$ GECAD Research Group, School of Engineering, Polytechnic of Porto, Porto, Portugal. ${ }^{2}$ Polytechnic of Porto, Porto, Portugal.

\section{Published: 10 October 2018}

\section{References}

Cheng R, Rodemann T, Fischer M, Olhofer M, Jin Y (2017) Evolutionary many-o bjective optimization of hybrid electric vehicle control: from general optimization to preference articulation. IEEE Trans Emerg Top Comput Intell 1(2):97-111

Di Somma M, Graditi G, Heydarian-Forushani E, Shafie-khah M, Siano P (2018) Stochastic optimal scheduling of distributed energy resources with renewables considering economic and environmental aspects. Renew Energy 116:272-287

Faia R, Pinto T, Vale Z, Corchado J (2017) An ad-hoc initial solution heuristic for metaheuristic optimization of energy market participation portfolios. Energies 10(7):883

Lezama F, Soares J, Hernandez-Leal P, Kaisers M, Pinto T, Almeida do Vale ZM (2018a) Local energy markets: paving the path towards fully Transactive energy systems. IEEE Trans Power Syst, in Press

Lezama F, Soares J, Vale Z, Rueda J (2017) Guidelines for the CEC'2018 competition on evolutionary computation in uncertain environments: a smart grid application

Lezama F, Soares J, Vale Z, Rueda J, Rivera S, Elrich I (2018b) 2017 IEEE competition on modern heuristic optimizers for smart grid operation: testbeds and results. Swarm Evol Comput, in Press

Soares J, Fotouhi Ghazvini MA, Silva M, Vale Z (2016) Multi-dimensional signaling method for population-based metaheuristics: solving the large-scale scheduling problem in smart grids. Swarm Evol Comput 29:13-32

Soares J, Pinto T, Lezama F, Morais H (2018) Survey on complex optimization and simulation for the new power systems paradigm. Complexity 2018:1-32

Talbi E-G (2009) Metaheuristics: from design to implementation

Yan Y, Qian Y, Sharif H, Tipper D (2013) A survey on smart grid communication infrastructures: motivations, requirements and challenges. IEEE Commun Surv Tutorials 15(1):5-20 